\title{
Dosimetry Impact of Boron and Its Carriers Structure at Boron Neutron Capture Therapy of a Brain Tumor; A Sim- ulation Study
}

\author{
Abbas Imani Pourya ${ }^{1}$, Ahmad Esmaili Torshabi ${ }^{1, *}$ \\ ${ }^{I}$ Faculty of Sciences and Modern Technologies, Graduate University of Ad- \\ vanced Technology, Kerman, Iran \\ *Corresponding author: Ahmad Esmaili Torshabi, Faculty of Sciences and \\ Modern Technologies, Graduate University of Advanced Technology, Haftbagh \\ Highway, Postal code: 7631818356, Kerman, Iran. Tel: +989386730223; Fax: \\ +983433776617; E-mail:ahmad4958@gmail.com; a.esmaili@kgut.ac.ir
}

DOI: $10.30699 /$ mci.5.3.479-1

Submitted: 10 November 2020

Revised: 28 April 2021

Accepted: 16 May 2021

e-Published: 30 June 2021

\section{Keywords:}

Boron Neutron Capture

Therapy

Boron

Drug Carriers

Radiation Dosage

Monte Carlo Method
Introduction: Boron neutron capture therapy (BNCT) is a method of cancer treatment and potentially, two borono-L-phenylalanine (BPA) and sodium borocaptate (BSH) are used in BNCT as common boron carriers. Some previous studies have shown that the dose rate is directly related to boron concentration in the tissue. This study aimed to simulate the structure of boron carriers and brain tumor compounds according to the real clinical condition. Moreover, the effect of carrier concentration in tumor volume and boron concentration in carriers' structure would be numerically assessed.

Methods: First, a phantom is developed by FLUKA simulation code to perform dosimetry aspects of Boron and carriers on a typical brain tumor. Moreover, the components of carriers were then simulated separately and the dosimetry parameters were assessed by changing the amount of boron in the carriers' structure and by the carriers' concentration variations inside and around the tumor to mimic the real condition.

Results: The results showed that by increasing boron inside the BPA structure from $5.17 \%$ to $50 \%$, the dose will be increased up to $78.46 \%$. In the BSH, tumor dose is raised to $9.06 \%$ by increasing the amount of boron concentration at carrier structure from $59 \%$ to $80 \%$. Moreover, by enhancing the two carriers' concentration inside the tumor volume up to $30 \%$, the dose value is increased up to $9.59 \%$ and $3.20 \%$ for BPA and BSH carriers, respectively.

Conclusions: The level of boron in the carrier's structure and also carriers' concentration is highly remarkable on dose delivery inside tumor volume and surrounding tissues.

C) 2021. Multidisciplinary Cancer Investigation

\section{INTRODUCTION}

Boron neutron capture therapy (BNCT) is known as a hopeful therapeutic strategy for treating tumor cells by selectively concentrating boron compounds inside tumor volume, and then its bombarding effect; using an epithermal neutron beam [1]. The thermal neutron capture cross-section of boron-10 is significantly high [2] and ${ }^{4} \mathrm{He}^{2+},{ }^{7} \mathrm{Li}^{3+}$, and low energy gamma radiation are accordingly produced after absorption of thermal neutrons $(\mathrm{E} \leq 0.4 \mathrm{eV})$ by boron-10. ${ }^{4} \mathrm{He}^{2+}$ and ${ }^{7} \mathrm{Li}^{3+}$ lose their kinetic energy by penetrating inside the tumor ( 4 to $9 \mu \mathrm{m}$ ) and cause damage to the tissues on their unique path [3-5]. The clinical and chemical studies of the BNCT have evolved since the early 1950s [6-9]. Many research efforts have been done to enhance the effectiveness of BNCT for cancer treatment. These studies have been conducted in various fields; ranging from neutron beam optimization to BNCT accessories 
and boron carriers $[10,11]$. At BNCT, carriers play an important role to transfer the boron element into the target volume. Potentially, there are two carriers of boron: 1) borono-L-phenylalanine (BPA) with the chemical formula $\mathrm{C}_{9} \mathrm{H}_{12} \mathrm{BNO}_{4}$ and 2) sodium borocaptate (BSH) with the chemical formula $\mathrm{Na}_{2} \mathrm{~B}_{12} \mathrm{H}_{11} \mathrm{SH}$; utilized as a commonly available carrier at BNCT. BPA and BSH are considerably accumulated inside the tumor, but a small fraction is also peripherally absorbed in the healthy surrounded tissues [12].

One of the challenging issues in increasing the BNCT efficiency is evaluating the effect of carrier concentration inside the tumor. Moreover, the level of the boron element in the chemical composition of the carrier drug is another issue that must quantitatively be taken into account. Therefore, the question arises as to dose fluctuation due to two following parameters: 1) the amount of boron in the carrier structure and 2) the amount of carrier in the tumor volume. According to the findings of some previous studies, the dose rate is directly related to the boron concentration in the tissue [13, 14]. But there is a lack of information concerning the components of BNCT, boron-included drugs, and carriers. In this study, the combination of tumor configured from cancerous tissue and boron carrier drugs have been considered similar to real conditions. For this aim, two BPA and BSH will be considered as boron carriers. This study yields a better understanding of the dose received by the tumor as a target by simulating various levels of boron concentration inside carriers and also carriers' concentration inside the target. It's worth mentioning that investigating the proposed strategy on boron and carriers concentration while implementing BNCT as a treatment modality is clinically impossible. However, the current study enables us to numerically realize it without any harm to patients.

To do this, the Monte Carlo FLUKA simulation code (Version 2011) was utilized as a verified common available code for simulating these parameters. FLUKA is a Monte Carlo-based simulation tool (developed by CERN in Switzerland and INFN in Italy) that simulates the transport of all kinds of particles in various fields [15]. The final analyzed results showed that with increasing boron concentration at drug structure, the absorbed dose inside tumor volume will be significantly enhanced. Moreover, the concentration of the carrier at the tumor site directly affects the dose enhancement at BNCT.

\section{METHODS}

In this work, the dosimetry impact of boron and its common available carriers has been evaluated as an analytical study. At BNCT, the carrier concentrations, as well as the amount of boron, are two effective parameters on the amount of dose, received by the tumor. These parameters have been considered in this study. To do this, a phantom of the human body was simulated with Monte Carlo FLUKA code, and then the effect of two parameters was investigated on a pre-defined brain tumor. FLUKA code is a multi-purpose verified simulation code that can calculate the interaction and propagation of various common available particles and photons at different matters with high accuracy. The valuable results of this code have made it highly applicable in different fields ranging from medical physics to dosimetry and shielding. It should be noted that the dimension of the phantom, material compounds information, and simulated organs and tumor have been defined according to the report of the International Commission on Radiological Protection (ICRP) [16, 17]. Two BPA and $\mathrm{BSH}$ as boron carriers have been considered during the simulation process. The BPA carrier is composed of $5.17 \%$ boron $\left({ }^{10} \mathrm{~B}\right), 5.79 \%$ Hydrogen $(\mathrm{H}), 51.72 \%$ Carbon $(\mathrm{C}), 6.70 \%$ Nitrogen $(\mathrm{N})$, and $30.62 \%$ Oxygen $(\mathrm{O})$. The percentage of chemical composition elements at $\mathrm{BSH}$ carrier is as follows: $59 \%$ boron $\left({ }^{10} \mathrm{~B}\right), 20.91 \%$ Sodium $(\mathrm{Na}), 5.5 \%$ Hydrogen $(\mathrm{H})$, and $14.58 \%$ Sulfur $(\mathrm{S})$. In the BNCT treatment strategy, using BPA and $\mathrm{BSH}$ as boron carriers is common mainly in the treatment of deep brain tumors when surgery and chemotherapy are not highly effective. Moreover, over the several past years many clinical studies have been done; using BPA and BSH carriers18] ]. Therefore, researchers have found the effects, side effects, and toxicity issues of these carriers. Figure 1 shows the brain tumor simulated in this study in the FLUKA geometry environment. Cancer cell components have been illustrated in Table 1. It has been attempted to simulate a Glioblastoma tumor located in the deep part of the brain. 


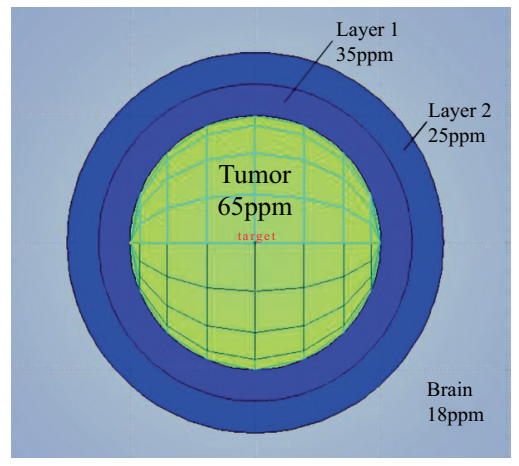

Figure 1: Boron Concentration at Brain Tumor, Two Outer Layers and Brain Tissue

The boron concentration is considered in the tumor central tissue, the layers around the tumor, and the brain

Table 1: Brain Tumor Compounds and Fractions Along With Two Inner and Outer Layers ${ }^{a}$

\begin{tabular}{lcccc}
\hline \multirow{2}{*}{ Organ } & \multicolumn{2}{c}{ Using BPA, \% } & \multicolumn{2}{c}{ Using BSH, \% } \\
\cline { 2 - 5 } & BPA & Brain Tissue & BSH & Brain Tissue \\
\hline $\begin{array}{l}\text { Tumor Central } \\
\text { Tissue }\end{array}$ & 0.1257 & 99.8743 & 0.011 & 99.989 \\
Layer 1 & 0.0676 & 99.9324 & 0.0059 & 99.9941 \\
Layer 2 & 0.0483 & 99.9517 & 0.0042 & 99.9958 \\
\hline
\end{tabular}

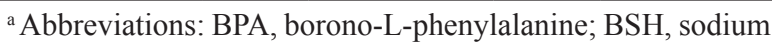
borocaptate

The tumor is located at a depth of $6.5 \mathrm{~cm}$ from the forehead and $5.5 \mathrm{~cm}$ from the top of the brain. After simulating the combinations of two BPA and BSH carriers, the tumor tissue warestimulated by adding each carrier, separately. Thus, the tumor tissue is composed of cancerous cells and carrier compounds. As shown in Figure 1 , the tumor volume consists of inner, middle, and outer parts. The inner part includes the cancerous tissues, and two outer layers are cancer-like tissues to mimic the real condition. This is because there is no sharp boundary between the carrier distribution inside the tumor volume and the lack of carrier at surrounding healthy tissues. Thus, it is assumed that the cancerous tissues are not completely dense and the concentration of the defined carriers does not sharply decrease to zero value in real clinical cases. Due to this, two layers around the cancerous tissues have been simulated to represent the soft reduced concentration of each carrier. After injecting carrier drug into the patient's body, it builds up throughout the patient's body while its accumulation in cancerous tissue is higher than healthy tissues. This point has also been considered during our simulation process. Due to this, boron concentrations have been softly reduced from $65 \mathrm{ppm}$ (at tumor volume) to 25-35 ppm (at two outer layers), and $18 \mathrm{ppm}$ (at normal brain tissue) to simulate actual condition $[12,14$, 19]. From the physical point of view, the boron level at the tumor site has a threshold to produce sufficient alpha particles as the main killers of cancerous cells after interacting (and then absorbing) with incidence neutrons; entered from outside of the patient body. Based on the former pieces of literature performed on the BNCT treatment modality, the minimum therapeutically effective concentration of boron is $35 \mathrm{ppm}$ inside the tumor [12]. Moreover, the maximum concentration of boron is not a fixed value and depends on various factors such as tumor type, tumor dimension, patient age, and so on. In a study performed by Koivunoro and colleagues, the maximum concentration of boron was found to be variable and up to $90 \mathrm{ppm}$ [14]. In our work, the boron concentration ranges from $65 \mathrm{ppm}$ to 85 $\mathrm{ppm}$ inside the tumor to be in an acceptable range and avoids possible serious toxicities [1, 14, 2022]. Moreover, by considering the structure of $\mathrm{BPA}$ and $\mathrm{BSH}$, and the amount of boron level in their structures, the carrier concentration inside the tumor should be $1257 \mathrm{ppm}$ for BPA and $110 \mathrm{ppm}$ for $\mathrm{BSH}$ to reach $65 \mathrm{ppm}$ of boron concentration inside the tumor. After simulating the phantom and its components, the dosimetry process is performed; using two separate carriers. The beam considered in this study has a radius of $1 \mathrm{~cm}$ according to the lateral size of tumor volume and consists of neutrons with $0.4 \mathrm{eV}$ energy. It is assumed that after neutron irradiation from outside of the body, they were converted into thermal neutrons in the tumor site to be absorbed by boron due to its high cross-section in colliding with neutrons. In this work, the boron concentration in its carrier structure has been changed as one of the challenging issues from a dosimetry point of view, since its experimental measurement is almost impossible on real patients. Using BPA carrier, boron concentration increased from $5.17 \%$ of the basic value to $10 \%, 20 \%, 30 \%, 40 \%$, and $50 \%$. Then, by using the BSH carrier, the boron level was increased from $59 \%$ of basic value to $70 \%$ and $80 \%$. At each stage of boron concentration, 
the maximum dose received by the tumor was numerically calculated; using simulation code. Apart from tumor dose, the above calculations have been done on two outer layers around the tumor volume and the maximum tumor dose was measured at each calculation step. The increment of carrier concentration in the components was $10 \%, 20 \%$, and $30 \%$, respectively (Table 2 ).

Table 2: The Changes in the Concentration of BPA and BSH Carriers in the Tumor Tissue, the Two Outer Layers Around the Tumor ${ }^{\mathrm{a}}$

\begin{tabular}{ccccccl}
\hline CI, \% & $\begin{array}{c}\text { CCT, } \\
\text { ppm }\end{array}$ & $\begin{array}{c}\text { CCL1, } \\
\text { ppm }\end{array}$ & $\begin{array}{c}\text { CCL2, } \\
\mathbf{p p m}\end{array}$ & $\begin{array}{c}\text { CCB, } \\
\text { ppm }\end{array}$ & $\begin{array}{c}\text { CBT, } \\
\text { ppm }\end{array}$ \\
\hline \multirow{4}{*}{ BPA } & 0 & 1257 & 676 & 486 & 348 & 65 \\
& 10 & 1383 & 744 & 531 & 383 & $\sim 71.5$ \\
& 20 & 1508 & 811 & 580 & 418 & $\sim 77.9$ \\
& 30 & 1634 & 879 & 628 & 452 & $\sim 84.44$ \\
\hline \multirow{4}{*}{ BSH } & 0 & 110 & 59 & 42 & 30 & 65 \\
& 10 & 121 & 65 & 46 & 33 & $\sim 71.5$ \\
& 20 & 132 & 71 & 50 & 36 & $\sim 77.9$ \\
& 30 & 143 & 77 & 55 & 39 & $\sim 84.44$ \\
\hline
\end{tabular}

${ }^{a}$ Abbreviations: BPA, borono-L-phenylalanine; BSH, sodium borocaptate; $\mathrm{CBT}$, concentration of the boron in the tumor; $\mathrm{CCB}$, concentration of the carrier in the brain; CCL1, concentration of carrier in layer 1; CCL2, concentration of carrier in layer 2; CCT, concentration of the carrier in the tumor; CI, carrier increase

\section{RESULTS}

The results showed that by increasing the amount of boron in the chemical structure of the carriers as a fraction, the maximum dose received by the tumor volume is increased accordingly. The results of this simulation are shown in Table 3 and Figure 2, as a quantitative assessment.

Table 3: Maximum Dose of the Tumor as a Function of boron Concentration (\%) in Two BPA and BSH Carriers Structure

\begin{tabular}{c|cc}
\hline & Boron in the Carrier, $\%$ & Max Tumor Dose Changes, $\%$ \\
\hline \multirow{4}{*}{ BPA } & 5.17 & 0 \\
& 10 & $\sim+13.93$ \\
& 20 & $\sim+31.76$ \\
& 30 & $\sim+50.15$ \\
& 40 & $\sim+68.76$ \\
\hline \multirow{2}{*}{ BSH } & 50 & $\sim+78.46$ \\
\hline & 59 & 0 \\
\hline
\end{tabular}

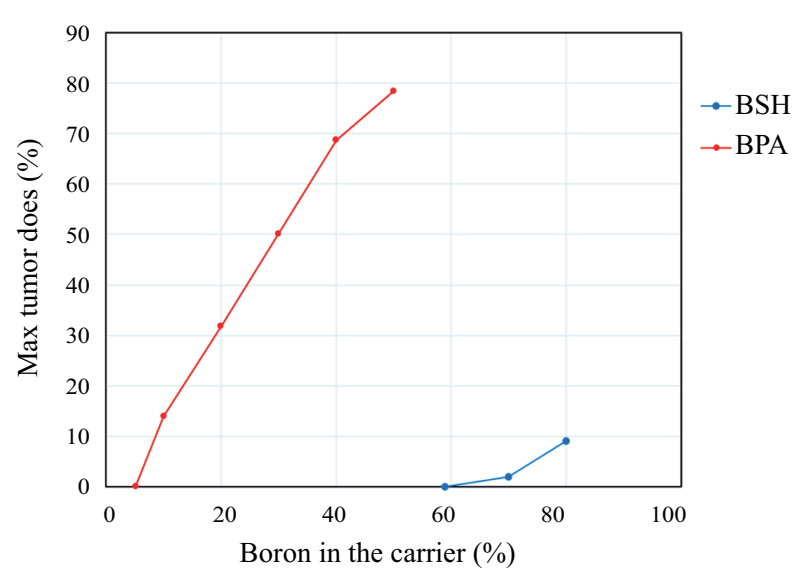

Figure 2: Changes in the Maximum Tumor Dose in Terms of Boron Presence at Both BPA and BSH Carriers

As depicted in Table 3 and Figure 2, by increasing boron in the structure of two carriers, the maximum dose received by the tumor is significantly increased. In BPA, tumor dose increased up to $78.46 \%$, by the amount of boron in the carrier reaching from $5.17 \%$ (as the base value) to $50 \%$. The same strategy was implemented; using $\mathrm{BSH}$ carrier and the tumor dose was increased up to $9.06 \%$, by enhancing the amount of boron from $59 \%$ (as the base value) to $80 \%$. It should be noted that the respective boron concentration in BPA and BSH is $5.17 \%$ and $59 \%$ in their initial form which was increased according to Table 3. It is concluded that with an increment in the amount of boron in the carrier structure, the boron concentration inside the tumor volume will be increased as well. Since the drug carrier accumulation is slightly different according to the patient's condition and type of drug structure, dosimetry was performed based on the changes in the concentration of carriers. The dosimetry process was performed separately for both the BPA and BSH carriers (Table 4 and Figure 3). Based on the results, the dose was increased by raising the carrier concentration in the tissues. According to Table 4 and Figure 3, the concentration of BPA carrier was increased from $1257 \mathrm{ppm}$ to $1634 \mathrm{ppm}$ (30\% increment percentage) and it was observed that the delivered dose onto tumor volume was then enhanced up to $9.59 \%$. In the BSH carrier, the carrier concentration was increased from $110 \mathrm{ppm}$ to $143 \mathrm{ppm}$ ( $30 \%$ increment percentage), and the dose reached to the tumor volume was observed to be increased up to $3.20 \%$. 
Table 4: Maximum Dose of the Tumor as a Function of Two BPA and BSH Carriers Concentration

\begin{tabular}{lll}
\hline & Carrier Concentration, $\%$ & Max Tumor Dose Changes, $\%$ \\
\hline BPA & 0 & 0 \\
& 10 & $\sim+6.34$ \\
& 20 & $\sim+7.62$ \\
& 30 & $\sim+9.59$ \\
\hline BSH & 0 & 0 \\
& 10 & $\sim+0.076$ \\
& 20 & $\sim+2.21$ \\
& 30 & $\sim+3.20$ \\
\hline
\end{tabular}

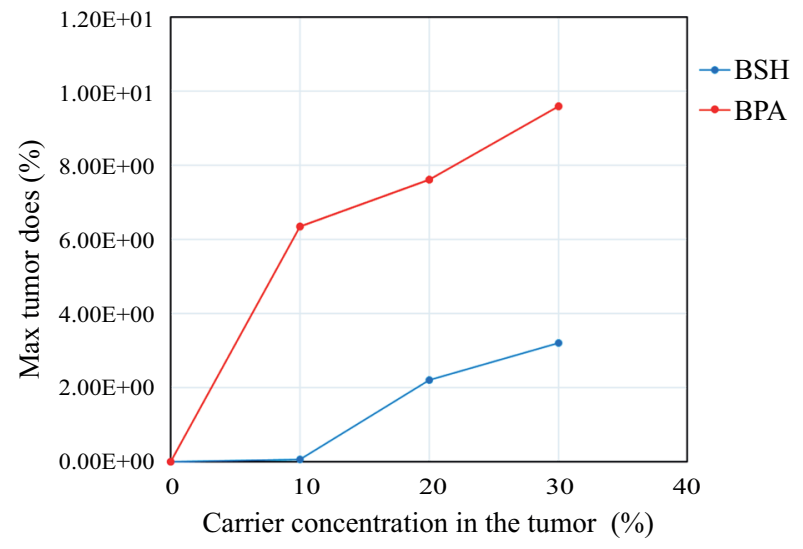

Figure 3: Changes in Maximum Tumor Dose in Terms of Carriers' Concentrations Enhancement

\section{DISCUSSION}

This study aimed to quantitatively evaluate the effect of boron and carriers parameters on the improvement of BNCT strategy for brain tumor treatment. Two main parameters as the amount of boron presence level in the carrier structure and carrier concentration in tumors and surrounding organs were taken into account and the role of each parameter on the delivered dose was quantitatively assessed. To do this, FLUKA Monte Carlo simulation code was utilized to simulate these parameters by defining brain tumor, neutron beam, required carriers, and all materials between the source and target at BNCT. An anthropomorphic phantom was simulated here to mimic the real condition of BCNT. To enhance the simulation results to reality, all simulation steps were defined according to medical sources and the report of the International Commission on Radiological Protection during simulation parameters definition. It should be considered that performing this investigation is almost impossible on real patients, while the simulation environment developed in this work enables us to numerically find dosimetry impacts of boron and carriers at the BNCT treatment method. The obtained results showed that by increasing the amount of boron in the structure of the carriers, the dose received by the tumor was increased. Because, with the increase of boron in the composition of the carrier drug, more borons will enter inside the cancerous tissue, and further ${ }^{10} \mathrm{~B}(\mathrm{n}, \alpha){ }^{7} \mathrm{Li}$ reaction will happen to kill cancerous cells. Therefore, each effort to make carrier drugs with a large number of borons in their structure is of great importance, because it can improve the BNCT cancer treatment strategy and ultimately leads to the development of cancer treatment. The results of this study showed that if the carrier concentration in the tumor and other components increases, the amount of tumor dose will also be enhanced. Considering this issue is important because the carrier's accumulation in the tumor can change. The cause of this change can be the biological condition of the patient's body, the type of drug structure, the amount of drug injection, or the invention of new methods of drug delivery. Thus, by increasing the carrier concentration in the tumor volume, the amount of boron also increases, and as a result, more ${ }^{10} \mathrm{~B}(\mathrm{n}, \alpha)^{7} \mathrm{Li}$ reactions occur that results in the dose enhancement.

On the other hand, it was found that in two tumors with the same condition, if BPA and BSH carriers were separately used, the boron concentration in the tumor reached $65 \mathrm{ppm}$. However, the maximum tumor dose using the BSH carrier would be slightly higher. It can be due to the different structure of the two carriers and the elements fraction in their chemical composition because different elements do not have the same capture cross-section. As shown in Figure 3, to investigate the effect of increasing BSH carrier concentration on tumor dose, the tumor dose was softly increased. However, with the use of BPA and increasing its concentration, the tumor dose was sharply increased. This indicates that the BSH carrier at high concentrations loses its advantage over the BPA carrier. It can therefore be suggested that BPA should be used when a high concentration of the carrier is required. When there is a need to use a low concentration of a carrier, the BSH carrier could be used which yields BNCT treatment efficiency. Furthermore, it should be noted that the concern may 
raise here about any possible toxicity due to boron concentration increment inside the patient body. Clinical signs of boron toxicity have been reported in the dose range of 100 to $55,500 \mathrm{mg}$ depending on age and body weight. For example, daily administration of $9000 \mathrm{ppm}$ of boron-containing compound $(68 \mathrm{mg}$ $\mathrm{B} / \mathrm{kg} / \mathrm{day}$ ) for 9 weeks, causes atrophy in rats [21] . On the other hand, the damage caused by boron toxicity in BNCT is less than the efficacy of this method as a cancer treatment modality. To increase the efficiency of the BNCT strategy, future studies may include further assessments on new carriers and also at the experimental phase.

In this investigation, dosimetry impacts of boron and its carriers at BNCT were comprehensively evaluated as a hopeful cancer treatment strategy. The main focus was on the effect of boron presence at the chemical composition of carriers and the concentration level of utilized carriers on the amount of dose received by tumor and surrounding healthy tissues. For this aim, FLUKA Monte Carlo simulation code was used to calculate the role of these parameters by defining an anthropomorphic phantom including brain tumor located at a specific location of the brain, boron variations, and two common available BPA and BSH carriers' concentration. Various concentrations of Boron and its carriers were considered to calculate the delivered dose at each simulation step. It's worth reporting that the delivered dose onto the tumor was increased by enhancing the presence of boron inside the carrier's structure and by enhancing carriers' concentration inside the tumor and nearby normal tissues. It should be considered that using boron by BPA and BSH carriers, like other drugs in high doses, can have clinical side effects. These side effects raise concerns while utilizing boron at high concentrations and frequently in a short period. On the other hand, the use of boron-included drugs is being done practically and their side effects must be in an acceptable range to be ignored in comparison with BNCT treatment benefits.

\section{ACKNOWLEDGMENTS}

Not applicable.

\section{CONFLICT OF INTEREST}

No conflict of interest applies to the work described in this manuscript.

\section{ETHICS APPROVAL}

Not applicable.

\section{REFERENCES}

1. Nedunchezhian K, Aswath N, Thiruppathy M, Thirugnanamurthy S. Boron Neutron Capture Therapy - A Literature Review. J Clin Diagn Res. 2016;10(12):ZE01-ZE4. DOI: 10.7860/JCDR/2016/19890.9024 PMID: 28209015.

2. Soloway AH, Barth RF, Carpenter DE. Advances in neutron capture therapy. Columbus, Ohio: Springer Science \& Business Media; 2012.

3. Hosmane NS, Maguire JA, Zhu Y, Takagaki M. Boron and Gadolinium Neutron Capture Therapy for Cancer Treatment. Singapore: World Scientific Publishing Co. Pte. Ltd.; 2012. DOI: $10.1142 / 9789814338684$ fmatter.

4. Yasui L, Kroc T, Gladden S, Andorf C, Bux S, Hosmane N. Boron neutron capture in prostate cancer cells. Appl Radiat Isot. 2012;70(1):6-12. DOI: 10.1016/j.apradiso.2011.07.001 PMID: 21784649.

5. Wang LW, Liu YH, Chou FI, Jiang SH. Clinical trials for treating recurrent head and neck cancer with boron neutron capture therapy using the Tsing-Hua Open Pool Reactor. Cancer Commun (Lond). 2018;38(1):37. DOI: 10.1186/ s40880-018-0295-y PMID: 29914577.

6. Wilkinson G, Stone FGA, Abel EW. Comprehensive organometallic chemistry. Oxford: Pergamon Press; 1982. DOI: 10.1002/ange.19830951031.

7. Schriver DF, Abel EW, Stone FGA, Bruce MI, Wilkinson G. Comprehensive organometallic chemistry II: a review of the literature 1982-1994. 1 ed. Oxford: Pergamon Press; 1995. DOI: 10.5860/choice.34-0311.

8. Mingos DMP, Crabtree RH. Comprehensive Organometallic Chemistry III. Netherlands: Elsevier; 2007.

9. Gielen M, Tiekink ERT. Metallotherapeutic drugs and metal-based diagnostic agents: the use of metals in medicine. USA: John Wiley \& Sons; 2005. DOI: 10.1002/0470864052.

10. Gabel D, Moss R, Alberts R. Boron neutron capture therapy: toward clinical trials of glioma treatment. Boston, USA: Springer; 1992. IX, 279 p. DOI: 10.1007/978-14615-3408-2.

11. Mishima Y. Cancer Neutron Capture Therapy. USA: Springer Science \& Business Media; 2013. XXVII, 920 p. DOI: $\quad 10.1007 / 978-1-4757-9567-7$.

12. Yokoyama K, Miyatake S, Kajimoto Y, Kawabata S, Doi A, Yoshida T, et al. Pharmacokinetic study of BSH and BPA in simultaneous use for BNCT. J Neurooncol. 2006;78(3):227-32. DOI: 10.1007/s11060-005-9099-4 PMID: 16557351.

13. Yu H, Tang X, Shu D, Geng C, Gong C, Hang S, et al. Impacts of multiple-field irradiation and boron concentration on the treatment of boron neutron capture therapy for nonsmall cell lung cancer. Int J Radiat Res. 2017;15(1):1-13. DOI: 10.18869 /acadpub.ijrr.15.1.1.

14. Koivunoro H, Hippelainen E, Auterinen I, Kankaanranta L, Kulvik M, Laakso J, et al. Biokinetic analysis of tissue 
boron $((1)(0) \mathrm{B})$ concentrations of glioma patients treated with BNCT in Finland. Appl Radiat Isot. 2015;106:189-94. DOI: 10.1016/j.apradiso.2015.08.014 PMID: 26363564.

15. Ferrari A, Sala PR, Fasso A, Ranft J. FLUKA : A multi-particle transport code (program version 2005). Geneva: CERN; 2005. 405 p. DOI: 10.5170/CERN-2005-010.

16. Snyder W, Cook M, Nasset E, Karhausen L, Howells GP, Tipton I. Report of the task group on reference man. Oxford: The International Commission on Radiotogical Protection 1975.

17. Standring S. Gray's Anatomy: The Anatomical Basis of Clinical Practice. Netherlands: Elsevier Limited; 2016. $1,562 \mathrm{p}$.

18. Kageji T, Mizobuchi Y, Nagahiro S, Nakagawa Y, Kumada H. Clinical results of boron neutron capture therapy (BNCT) for glioblastoma. Appl Radiat Isot. 2011;69(12):1823-5. DOI: 10.1016/j.apradiso.2011.05.029 PMID: 21684170.

19. Chou FI, Chung HP, Liu HM, Chi CW, Lui WY. Suitability of boron carriers for BNCT: accumulation of boron in malignant and normal liver cells after treatment with BPA, BSH and BA. Appl Radiat Isot. 2009;67(7-8 Suppl):S105-8. DOI: 10.1016/j.apradiso.2009.03.025 PMID: 19375330 .

20. Farfan-Garcia ED, Castillo-Mendieta NT, Cipres-Flores FJ, Padilla M, II, Trujillo-Ferrara JG, Soriano-Ursua MA. Current data regarding the structure-toxicity relationship of boron-containing compounds. Toxicol Lett. 2016;258:11525. DOI: 10.1016/j.toxlet.2016.06.018 PMID: 27329537.

21. Bakirdere S, Orenay S, Korkmaz M. Effect of boron on human health. Open Miner Process J. 2010;3(1):54-9. DOI: 10.2174/1874841401003010054.

22. Mehta SC, Lu DR. Interspecies pharmacokinetic scaling of BSH in mice, rats, rabbits, and humans. Biopharm Drug Dispos. 1995;16(9):735-44. DOI: 10.1002/ bdd.2510160904 PMID: 8580398.

bbits, and humans. Biopharm Drug Dispos. 1995;16(9):735-44. http://dx.doi.org/10.1002/bdd.2510160904 http://www. ncbi.nlm.nih.gov/pubmed/8580398. 